\title{
Accelerated versus conventional Ponseti protocol for the treatment of idiopathic talipes equinovarus deformity: A short term follow up in Iraq
}

\begin{abstract}
Background and objective: The accelerated protocol of Ponseti method was suggested to shorten the period of treatment of the conventional one for the cases of talipes equinovarus deformity. This study aimed to compare the accelerated protocol of Ponseti method in the treatment of clubfoot deformity with the conventional one.
\end{abstract}

Methods: A prospective comparative study was conducted for infants less than six months with congenital talipes equinovarus deformity. The patients were randomized to either Group 1 (casts changed every week, conventional protocol of Ponseti method) or Group 2 (twice weekly, accelerated one). Pirani score was used to assess the severity of the deformity at presentation, at time of last cast removal, and at the last follow up visit (6 $6^{\text {th }}$ months).

Results: The patients included were 48 cases with 79 feet. Group 1 (39 clubfeet) had a mean Pirani score of $5.6( \pm 1.15)$ at presentation, which dropped to $0.47( \pm 0.41)$ when the last cast was removed. In Group 2 (40 clubfeet), it dropped from 5.57 ( \pm 0.83 ) to 0.77 $( \pm 0.01)$. The result of each treatment protocol was significant, but the difference between them was not significant. Five cases (three patients aged more than three months) of Group 2 needed eight casts to reach an acceptable position of correction. The difference between the mean number of casts applied in Group 1 (5.09) and Group 2 (5.82) was statistically not significant. However, the difference between the mean number of days spent in the cast was significant. The complications occurred in 12 out of 79 feet, with no statistically significant difference between both groups.

Conclusion: The accelerated protocol of Ponseti method for treating clubfoot deformity is as effective and as safe as the conventional one. It shortens the time required to complete the treatment program. Those who present lately (beyond the age of three months) may require an additional number of casts.

Keywords: Clubfoot; Congenital talipes equinovarus; Ponseti; Accelerated; Cast.

\section{Introduction}

Clubfoot is a birth defect where one or both feet are rotated inward and downward. Without treatment, people walk on the sides of their feet, which causes problems with walking. It occurs in about 1 per 1000 newborns. The idiopathic form (also known as congenital talipes equinovarus deformity [CTEV]) is more common than the other secondary forms like neurogenic and syndromic ones. It is a complex deformity that involves pathological anatomy, including ankle equinus, with hindfoot varus, midfoot cavus, and forefoot adductus. $^{1,2}$ The Ponseti method for the treatment of children with CTEV gained wide acceptance in the worldwide orthopedic community in the last few decades. The method is well established and has been shown to be highly effective with good long-term results. The conventional protocol of this treatment method includes gradual correction of the deformity using sequences of

${ }^{1}$ Department of Surgery, Medical College, University of Duhok, Duhok, I raq.

2 Department of Orthopaedics, Hawler Teaching Hospital, Erbil, I raq.

* Correspondence: jagaromar@uod.ac 
manipulations and plaster casting on a weekly interval base. This serial casting usually includes 4-6 casts on average over 4-6 weeks. Once corrected, the position of the corrected foot is maintained by the feet abduction brace until the age of four years to prevent relapse. ${ }^{3-7}$ The accelerated protocol of Ponseti method was suggested to shorten the period of treatment of the conventional one. The fundamental of manipulations and plaster casting remains the same but is changed twice or three times per week. ${ }^{8-14}$ This study aimed to determine the effectiveness of the accelerated Ponseti method in the treatment of cases with clubfoot deformity and compare it with conventional one in Iraq.

\section{Methods}

The study was a prospective comparative one. The approval to conduct this study was obtained from the Surgical Specialties' Council of Kurdistan Board of Medical Specialties. Infants in their first year of life who were born with the CTEV deformity in one or both of their feet were selected for this study. The patients were selected from those who attained the following medical places for management from January till September 2019; Early Detection of Childhood Disability Center at Duhok city, Emergency Teaching Hospital in Duhok city, Helina Center for Handicapped Children in Erbil city and Hawler Teaching Hospital in Erbil city. The inclusion criteria were children with idiopathic clubfoot (CTEV) deformity in one or both of their feet, the Pirani score was more than one at presentation, age less than six months, and had not been treated previously by any method. The exclusion criteria were children with clubfoot deformity due to a neurogenic disorder (like spina bifida) or part of a syndromic disorder (like arthrogryposis, tibial hemimelia, or constricting congenital bands), those who were previously treated specially by surgery, or those who had a postural clubfoot deformity (Pirani score of one or less). The patients were randomized by the authors to either the standard protocol (Group 1) or the accelerated one (Group 2) of Ponseti method in an alternating consecutive manner. The first case was included in Group 1, the second case was included in Group 2, the third case was included in Group 1, and so on). Randomization occurred at the patient level, with both feet being treated using the same method in case of bilateral feet deformity. Informed consent from the patient's parents was obtained before starting the treatment. The data of each patient was reported in a special file which was used later on during the program of treatment and for follow up later on. The severity of the deformity of each foot was assessed by Pirani score system for clubfoot at presentation before starting treatment, during each session of manipulation and cast changes, at the time of last cast removal, and at the last follow up visit (around the sixth months after the removal of the last cast). Each case of clubfoot was treated in the classical way according to Ponseti principles for the treatment of clubfoot. A percutaneous tendo Achillis tenotomy was performed if dorsiflexion was $<10^{\circ}$ at the end of the last manipulation and casting. The casts were changed every week in patients included in Group 1 (conventional Ponseti protocol) and twice weekly for those included in Group 2 (accelerated Ponseti protocol). The last cast was maintained for three weeks in all cases regardless of the group they belong. The number of casts and the number of days (from the day of the first cast till the day of the last cast application) were reported for each case. After removal of the last cast, the Pirani score was assessed, and then the feet of the patient of either group were placed in a foot abduction brace to maintain the corrected position of the foot with follow up at two months intervals. The Pirani score at the sixth month of follow up was assessed, and any relapse of any component of the deformity was reported in the data 
https:// doi.org/ 10.15218/ zjms.2021.009

file of the patient (Figure 1). The data were collected and analyzed statistically using the statistical package for the social sciences software program (version 19). The mean Pirani score at presentation and at the time of removal of the last cast was used in the analysis (by using the paired t-test) for evaluation of the effectiveness of each protocol in the treatment of clubfoot deformity. To compare between both groups, the mean difference of Pirani scores, the mean number of casts applied, the mean number of days spent in casts (from first cast day till the day of last cast application), and the number of complications (including relapses) of the cases of each group were included. These comparison processes were done statistically by using the independent t-test to find the $P$ value. When the expected value of more than $20 \%$ of the cells of the table was less than 5 , then the $P$ value was founded by the Fisher's Exact test. A $P$ value of $<0.05$ was considered statistically significant.

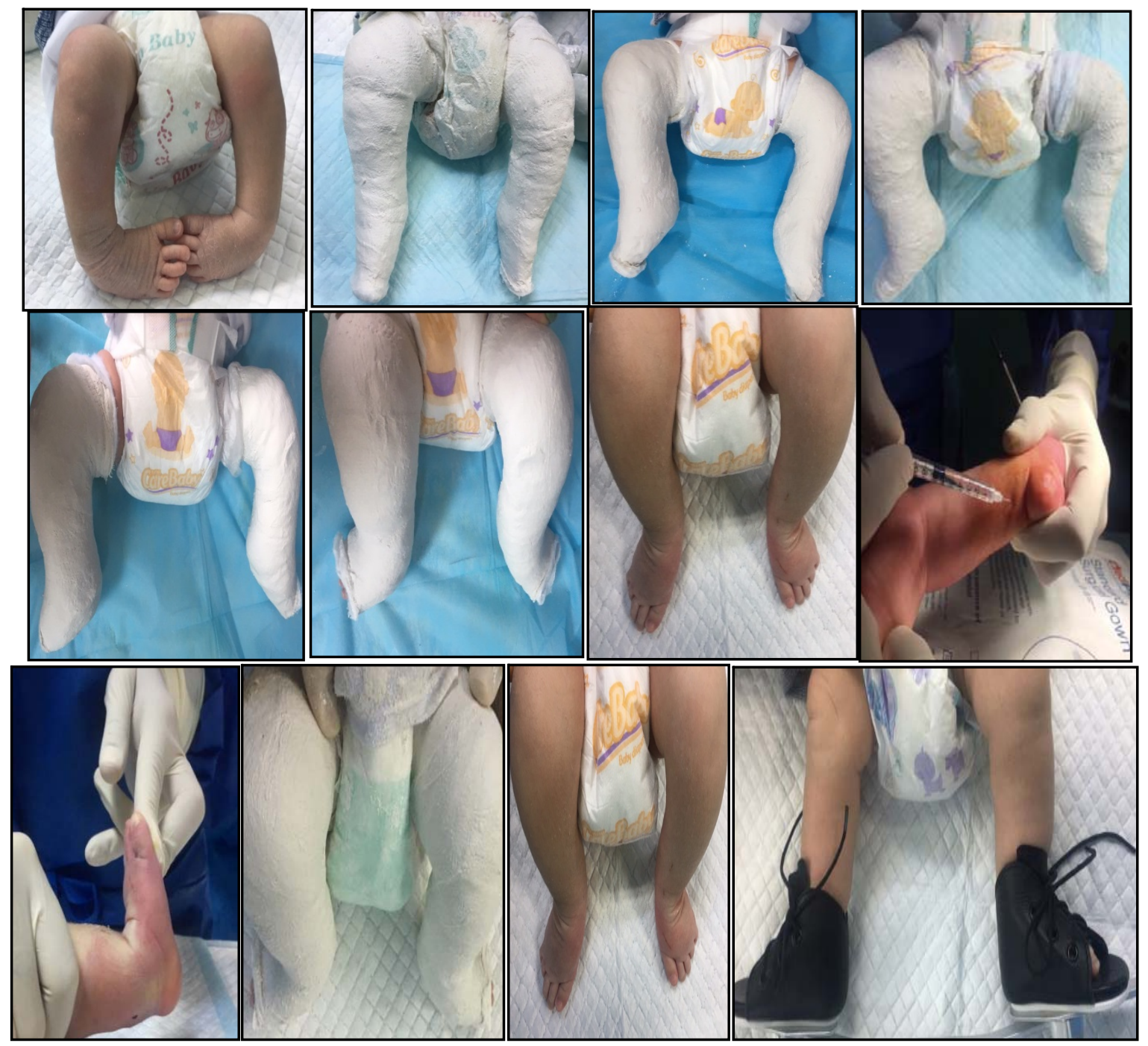

Figure 1: Steps of Ponseti method in both accelerated and conventional protocol. 


\section{Results}

The patients included in this study were 48 cases; 32 (66.7\%) males and 16 (33.3\%) females. The male to female ratio was $2: 1$. The mean age of the patients at presentation and beginning of treatment was 15.09 days with a standard deviation of 19.58. Those included in Group 1 (treated according to the conventional protocol of Ponseti method) were 23 children with 39 clubfeet (16 cases had bilateral feet deformities). In Group 2 (treated by the accelerated protocol), 15 out of 25 cases had bilateral feet deformities (total 40 feet). So, the total number of feet treated was 79 feet (39 by the conventional protocol and 40 by the accelerated one). The mean Pirani score ). for the patients of Group 1 at presentation was 5.6 ( \pm 1.15 standard deviation). It dropped to $0.47( \pm 0.41)$. The statistical analysis of this result was significant $(P<0.001)$. However, the Pirani score raised slightly six months later because some cases showed some evidence of relapses. The same statistically significant $(P<0.001)$ gained during checking the results of the patients of Group 2. The difference between both groups was statistically not significant $(P=0.5283)$ (Table 1). Some cases of this group also showed mild degrees of relapse, which were comparable with those of Group 1, who also developed relapse of the deformity (Figure 2).

Table 1: Differences in Pirani score between both groups.

\begin{tabular}{lcccc}
\hline & $\begin{array}{c}\text { Pirani score at } \\
\text { presentation }\end{array}$ & $\begin{array}{c}\text { Pirani score at } \\
\text { the time of last } \\
\text { cast removal }\end{array}$ & $\begin{array}{c}\text { Mean differences } \\
\left( \pm \mathrm{SD}^{*}\right)\end{array}$ & $\boldsymbol{P \text { value }}$ \\
\hline Group 1 $(\mathrm{n}=39)$ & $5.6( \pm 1.15)$ & $0.47( \pm 0.41)$ & $5.55( \pm 0.74)$ & $<0.001$ \\
Group 2 $(\mathrm{n}=40)$ & $5.57( \pm 0.83)$ & $0.77( \pm 0.01)$ & $4.8( \pm 0.82)$ & $<0.001$ \\
\hline
\end{tabular}

*SD: Standard Deviation

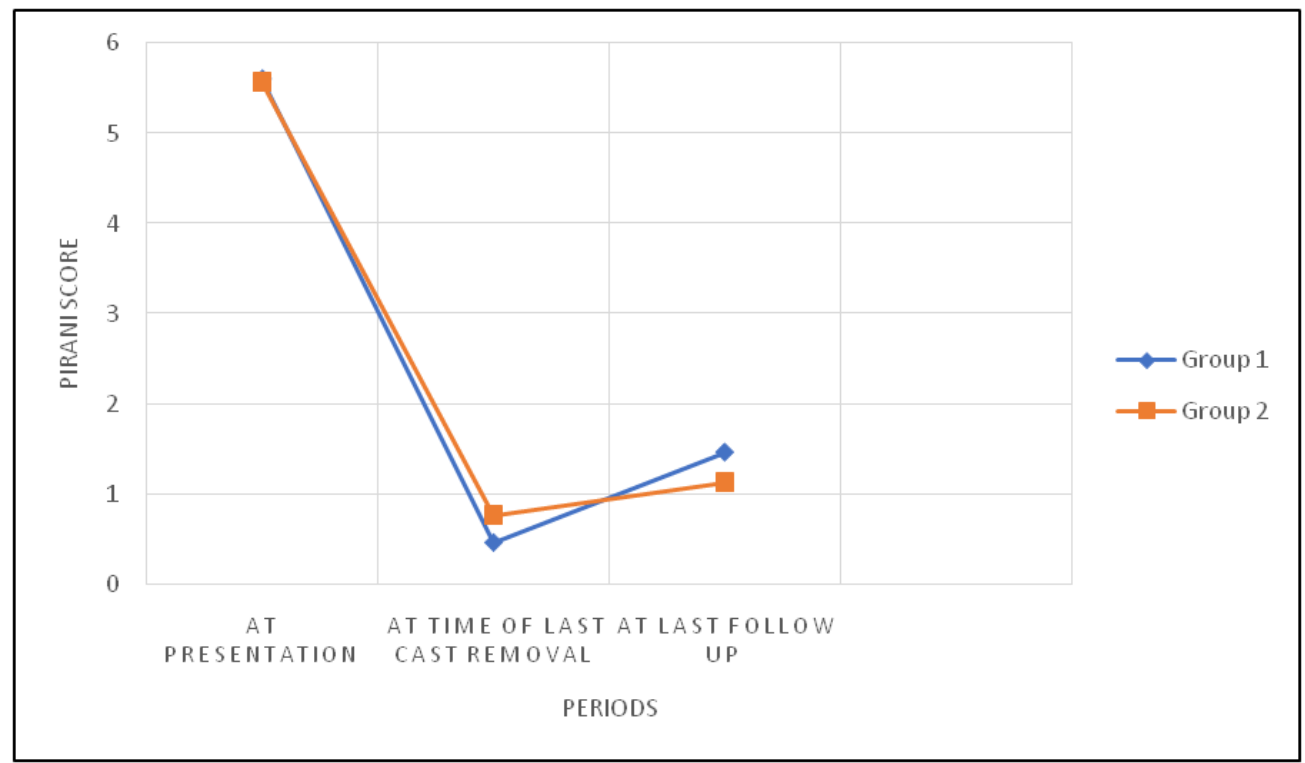

Figure 2: Pirani score of both groups per periods. 
The mean number of casts applied in Group 1 was 5.09 and in Group 2 was 5.82. The difference between both groups was statistically not significant. Five cases (3 patients) of Group 2 needed eight casts in order to fully correct their feet deformities. They were all more than three months of their age when they present and started their treatment. Four cases (two patients) of Group 2 also were more than three months of age at presentation, but their treatment was completed by six casts. Regarding the mean number of days that each group spent in cast between the first one and the application of the last one, the difference between both groups was statistically significant $(P<0.001)$ (Table 2$)$. The tendo Achillis tenotomy was done in all cases except two cases of Group 1 and one case of Group 2. The complications occurred in 12 (15.19\%) out of 79 feet. The skin sores occurred in $3(3.79 \%)$ cases and relapse of the deformity in 9 (11.39\%) cases (Table 3). These complications occurred in both groups with no statistically significant difference between them $(P=0.634)$. Five patients who developed relapse required surgical correction later on in the form of postero-medial release.

Table 2: Differences between both groups regarding the number of casts and days in the cast.

\begin{tabular}{lccc}
\hline Variable & $\begin{array}{c}\text { Group 1 } \\
\text { Mean }\left( \pm \text { SD}^{*}\right)\end{array}$ & $\begin{array}{c}\text { Group 2 } \\
\text { Mean }( \pm \text { SD*) }\end{array}$ & P value \\
\hline Number of casts & $5.09( \pm 0.59)$ & $5.82( \pm 0.61)$ & 0.84 \\
Number of days in casts & $35.63( \pm 5.1)$ & $19.37( \pm 12.6)$ & $<0.001$ \\
\hline
\end{tabular}

*SD: Standard Deviation

Table 3: Complications occurred in both groups.

\begin{tabular}{ccccc}
\hline Complication & Group 1 (n=39) & Group 2 (n=40) & Total $(\mathbf{n = 7 9 )}$ & $\boldsymbol{P}$ value \\
\hline Skin complications & $1(2.56 \%)$ & $2(5 \%)$ & $3(3.79 \%)$ & 0.212 \\
Relapse & $5(12.82 \%)$ & $4(10 \%)$ & $9(11.39 \%)$ & 0.422 \\
Total & $6(15.38 \%)$ & $6(15 \%)$ & $12(15.19 \%)$ & 0.634 \\
\hline
\end{tabular}




\section{Discussion}

The Ponseti technique is well established, and its conventional protocol (which depends on weekly changing the cast) has been shown to be highly effective., However, in certain conditions (as in some developing countries), it may be difficult for some families to travel for long distance every week for cast changes. It may also be difficult to maintain the cast clean till the next appointment. Hence, the idea of the accelerated protocol of Ponseti method came to solve these problems. ${ }^{8,9,12,13,15}$ The results of the present study showed that both protocols of Ponseti method of treatment were effective in the treatment of clubfeet deformity as the Pirani scores of both groups drops to an acceptable level at the end of the casting step. Also, there was no statistical difference between both of them. This result supports the results of other studies ${ }^{8,10,13-17}$ and it can be concluded that the accelerated protocol is as effective as the conventional one. The mean number of the casts applied for both groups did not significantly differ, but the mean number of days spent in the cast did that. This was the main advantage of the accelerated protocol. It can be considered important for some families who can not travel frequently and long distances to complete the treatment program. It was interesting to notice that some of the cases of Group 2, who were more than three months of age at presentation, required more than the usual number of casts (eight casts with a duration of 28 days) in order to reach an acceptable position of correction. The authors of the study suspect that the progressive tightness of the postero-medial structures with growing age may cause that. These structures need more time to regenerate and elongate under the stretching stimulus. As the number of casts increases, the number of days spent in the cast also increases. But still, the difference was significant regarding the number of days required to complete the treatment program. So, it can be concluded that even if the patient presents late (age more than three months), still the accelerated protocol can be applied, but with suspected a greater number of casts required. The mean number of complications for the patients included in this study was relatively low compared to the results of other studies. ${ }^{8,10,16}$ The difference between both groups regarding the mean number of complications that occurred was statistically not significant. Therefore, it can be concluded that the accelerated protocol was as safe as the conventional one. The limitation of this study was the short term follow of patients since six months of follow up is considered a short term follow up for such cases. The preferable follow up maybe four years, and a new study has recommended that. In a study done in India, the researchers evaluated the efficacy of the accelerated Ponseti technique in the management of CTEV deformity cases. They concluded at five years of follow up that there was no significant difference in the functional outcome between the standard and the accelerated protocol. ${ }^{17}$

\section{Conclusion}

The accelerated protocol of Ponseti method for treating clubfoot deformity is as effective and as safe as the conventional one. It shortens the time required to complete the treatment program. Those who present lately (beyond the age of three months) may require an additional number of casts. A new study is recommended for follow up of these cases for a longer term.

\section{Competing interests}

The authors declare no competing interests.

\section{References}

1. Gibbons PJ, Gray K. Update on clubfoot. J Paediatr Child Health 2013; 49(9):E434-7.

2. Dobbs MB, Gurnett CA. Update on clubfoot: etiology and treatment. Clin Orthop Relat Res 2009; 467(5):1146-53.

3. Morcuende JA, Dolan LA, Dietz FR, Ponseti IV. Radical reduction in the rate of extensive 
corrective surgery for clubfoot using the Ponseti method. Pediatrics 2004; 113:376-80.

4. Herzenberg JE, Radler C, Bor N. Ponseti versus traditional methods of casting for idiopathic clubfoot. J Pediatr Orthop 2002; 22:517-21.

5. MacNicol M. The management of club foot: issues for debate. J Bone Joint Surg Br 2003; 85-B:16770.

6. Zwick EB, Kraus T, Maizen C, Steinwender G, Linhart WE. Comparison of Ponsetiversus surgical treatment for idiopathic clubfoot: a shortterm preliminary report. Clin Orthop Relat Res 2009; 467(10):2668-76

7. Siapkara A, Duncan R. Congenital talipes equinovarus: A review of current management. J Bone Joint Surg Br 2007; 89:995-1000.

8. Harnett P, Freeman R, Harrison WJ, Brown LC, Beckles V. An accelerated Ponseti versus the standard Ponseti method: A prospective randomized controlled trial. J Bone Joint Surg $\mathrm{Br}$ 2011; 93:404-8.

9. Sharma P, Yadav V, Verma R, Gohiya A, Gaur S. Comparative analysis of results between conventional and accelerated Ponseti technique for idiopathic congenital clubfoot. Orthop J MP 2016; 22:3-7.

10. Morcuende JA, Abbasi D, Dolan LA, Ponseti IV. Results of an accelerated Ponseti protocol for clubfoot. J Pediatr Orthop 2005; 25:623-6.

11. Ullah S, Inam M, Arif M. Clubfoot management by accelerated Ponseti technique. RMJ 2014; 39:418-20.

12. Sutcliffe A,Vaea K, Poulivaati J, Evans AM. 'Fastcasts':Evidence based and clinical considerations for rapid Ponseti method. Foot Ankle Online J 2013; 6:2.

13. Solanki M, Ajmera A, Rawat S. Comparative study of accelerated Ponseti method versus standard Ponseti method for the treatment of idiopathic clubfoot. J Orthop Traumatol Rehabil 2018; 10:116-9.

14. Sahu B, Rajavelu R, Tudu B. Management of idiopathic congenital talipes equinovarus by standard versus accelerated Ponseti plaster technique: A prospective study. J Orthop Traumatol Rehabil 2015; 8:30-4.

15. Ahmad AA, Aker L. Accelerated Ponseti method: First experiences in a more convenient technique for patients with severe idiopathic club feet. Foot Ankle Surg 2019; 26(3):254-57.

16. Barik S, Nazeer M, Mani BT. Accelerated Ponseti technique: Efficacy in the management of CTEV. Eur J Orthop Surg Traumatol 2019; 29(4):91924.

17. Elgohary HS, Abulsaad M. Traditional and accelerated Ponseti technique: A comparative study. Eur J Orthop Surg Traumatol 2015; 25(5):949-53. 